\title{
Oral chemotherapy safety practices at US cancer centres: questionnaire survey
}

\author{
Saul N Weingart, vice president for patient safety ${ }^{1}$, Jonathan Flug, medical student ${ }^{2}$, Daniela \\ Brouillard, administrative assistant', Laurinda Morway, research coordinator', Ann Partridge, staff \\ physician ${ }^{3}$, Sylvia Bartel, pharmacy director ${ }^{3}$, Lawrence N Shulman, chief medical officer ${ }^{3}$, Maureen \\ Connor, vice president for quality improvement and risk management ${ }^{3}$
}

\section{ABSTRACT}

Objective To characterise current safety practices for the use of oral chemotherapy.

Design Written questionnaire survey of pharmacy

directors of cancer centres.

Setting Comprehensive cancer centres in the United States.

Results Respondents from 42 (78\%) of 54 eligible centres completed the survey, after consulting with 89 colleagues. Clinicians at 29 centres used handwritten prescriptions, two used preprinted paper prescriptions, and six used electronic systems for most oral chemotherapy prescribing. For six commonly used oral chemotherapies, on average 10 centres required a diagnosis on the prescription, 11 required the protocol number, four required the cycle number, nine required double checking by a second clinician, 14 required a calculation of body surface area, and 14 required a calculation of dose per square metre of body surface area. Only a third of centres requested patients' written informed consent when oral chemotherapy was given off protocol. Nearly a quarter (10) of centres had no formal process for monitoring patients' adherence. In the past year respondents at 10 centres reported at least one serious adverse drug event related to oral chemotherapy, and respondents at 13 centres reported a serious near miss.

Conclusion Few of the safeguards routinely used for infusion chemotherapy have been adopted for oral chemotherapy at US cancer centres. There is currently no consensus at these centres about safe medication practices for oral chemotherapy.

\section{INTRODUCTION}

Common malignancies can be treated with oral chemotherapy. ${ }^{1}$ This offers patients unprecedented convenience compared with intravenous infusion therapy. ${ }^{2-4}$ Given the potential toxicities of oral chemotherapy and the importance of adherence for successful treatment, ensuring safe use of these drugs may require special safeguards. ${ }^{4-11}$ The extent to which such safety practices have been introduced into clinical care is unknown.
To characterise current safety practices for oral chemotherapy we surveyed pharmacy leaders at comprehensive cancer centres in the United States. Given the relatively recent introduction of oral chemotherapies, we expected considerable variation in practice.

\section{METHODS}

Sample selection-We used the internet to compile contact information for pharmacy directors at all 62 comprehensive cancer centres designated by the US National Cancer Institute. We excluded eight research centres that do not directly care for patients.

Instrument development -We developed a questionnaire that examined aspects of the use of oral chemotherapy medication. We focused on non-hormonal oral agents with risk of serious toxicity including capecitabine, cyclophosphamide, gefitinib, imatinib, oral methotrexate, and temozolomide. We circulated a draft survey among the quality directors of the Comprehensive Cancer Center Consortium for Quality Improvement and incorporated revisions into the final instrument.

Study protocol - We sent the questionnaire by email and post in September 2005. Non-responders received up to three follow-up telephone calls. If the pharmacy director was misidentified or unable to participate, we encouraged recipients to identify a substitute respondent who was familiar with oral chemotherapy practices.

Data analysis-We deleted respondents' names and organisations before analysis and tabulated responses using Stata 7.0 (StataCorp, College Station, TX).

\section{RESULTS}

Responses

We received 42 completed questionnaires from the 54 eligible centres. Table 1 shows the job titles of 33 respondents who reported this information. Thirty one respondents consulted with colleagues about the survey (total 89, median 2, range 0-8).

Prescribing

Prescribing practices for oral chemotherapy varied considerably. For most oral chemotherapy prescribing 
clinicians at 29 centres used handwritten prescriptions. At two centres they used preprinted paper prescriptions and at six they used electronic systems (table 2).

Organisations did not have many compulsory requirements on prescriptions. For six commonly used oral chemotherapies (see bmj.com), on average 10 centres required a diagnosis on the prescription, 11 required the protocol number (when appropriate), four required the cycle number, nine required double checking by a second clinician, 14 required a calculation of body surface area, and 14 required calculation of dose per square metre of body surface area. Over half (23) had no required element for oral chemotherapy prescriptions.

Lack of standardisation was also reflected in the use of informed consent. Only a third of centres asked patients to provide written informed consent when oral chemotherapy was given off protocol.

\section{Coordination and monitoring}

Respondents reported considerable variation in the methods used to coordinate oral and infusion chemotherapy among patients who received both types of treatment. Twenty five centres coordinated care by maintaining a record of oral chemotherapy in the patient's medication profile. At 26 centres, a nurse or pharmacist reviewed oral chemotherapy during infusion treatment. Seven centres reported no formal coordination.

Clinicians at most centres monitored patients' adherence to oral chemotherapy during office visits. Staff at 10 centres asked patients to bring in logbooks, and staff at nine regularly counted pills. Respondents from nine centres reported no formal process for monitoring adherence.

\section{Pharmacy practices}

Pharmacy services may be underused in the care of patients on oral chemotherapy. Although 33 centres offered patients a formal consultation with a

Table 1| Details of 42 respondents from US cancer centres who took part in survey of safety practices with oral chemotherapy

\begin{tabular}{lc} 
& No \\
\hline Job title given by respondent: & \\
\hline Pharmacy director, manager, supervisor & 19 \\
\hline Oncology pharmacist & 8 \\
\hline Quality or clinical outcomes manager & 3 \\
\hline Clinical nurse specialist & 2 \\
\hline Physician director of clinical services & 1 \\
\hline Not specified & 9 \\
\hline Colleagues (n=89) with whom respondent discussed survey: & \\
\hline Pharmacist & 28 \\
\hline Pharmacy director or manager & 19 \\
\hline Nurse & 13 \\
\hline Nurse manager or administrator & 10 \\
\hline Nurse practitioner & 6 \\
\hline Attending physician & 5 \\
\hline Quality, risk, or safety director & 5 \\
\hline Clinical fellow & 2 \\
\hline Medical director or chief medical officer & 1 \\
\hline
\end{tabular}

pharmacist, respondents estimated that many $(42 \%)$ patients declined a consultation.

Thirty four centres had an on-site pharmacy for patients receiving oral chemotherapy who were not on research protocols. Respondents estimated, however, that $38 \%$ of eligible patients did not use this facility. Failure to use an on-site pharmacy may be problematic as respondents at 17 centres rated communication between community pharmacies and cancer centres as fair or poor.

\section{Education of patients}

At most centres physicians shared responsibility with other health professionals for educating patients about oral chemotherapy. Respondents at 25 (60\%), 34 $(81 \%)$, and $40(95 \%)$ organisations indicated that nurse practitioners, nurses, and pharmacists, respectively, were also responsible for educating patients about use and safety. Only a third of organisations provided special training or certification for those who educate patients about these medications.

\section{Safety assessment}

Respondents at 10 centres reported that a "serious adverse drug event" related to oral chemotherapy had occurred in the past year, and 13 centres reported a "serious near miss." Respondents at 36 centres indicated that clinicians in their organisations were concerned about the risks of oral chemotherapy.

\section{DISCUSSION}

Despite the increased use of oral chemotherapy, current practices for prescribing, coordinating and monitoring, and dispensing these medications and educating patients in US cancer centres leave room for improvement. We found that most organisations had no required elements for prescribing oral chemotherapy and few requested patients' written informed consent for off protocol prescribing. Only about half of cancer centres coordinated oral with intravenous chemotherapy. Most organisations provided little infrastructure to support adherence to treatment. On-site pharmacies and consultation with a pharmacist were widely available to patients, but both were underused. Clinicians from various professions shared responsibility for educating patients about oral chemotherapy, but few centres provided clinicians with relevant formal training.

Table 2 Prescription writing for oral chemotherapy at 42 US cancer centres. Figures are numbers (percentages) of centres

\begin{tabular}{|c|c|c|}
\hline & \multicolumn{2}{|c|}{$\begin{array}{l}\text { Proportion of prescriptions generated by } \\
\text { method }\end{array}$} \\
\hline & Majority (> 50\%) & Minority $(\leq 50 \%)$ \\
\hline $\begin{array}{l}\text { Handwritten paper } \\
\text { prescriptions }\end{array}$ & $29(69)$ & $13(31)$ \\
\hline $\begin{array}{l}\text { Preprinted paper } \\
\text { prescriptions }\end{array}$ & $2(5)$ & $40(95)$ \\
\hline Electronic order entry & $6(14)$ & $36(86)$ \\
\hline
\end{tabular}




\section{WHAT IS ALREADY KNOWN ON THIS TOPIC}

Although oncologists prescribe oral chemotherapy for many indications, little is known about associated safety practices

\section{WHAT THIS STUDY ADDS}

Few of the safeguards in routine use for infusion chemotherapy have been adopted for oral chemotherapy chemotherapies, there is no apparent consensus among oncology professionals about safe practices for these drugs. Safeguards used for infusion chemotherapy cannot be abandoned for oral treatment. The oncology community must define safe medication practices appropriate for oral chemotherapy, develop practice guidelines, and accelerate their adoption.

We thank the Comprehensive Cancer Center Consortium for Quality Improvement (C4QI) for help in completing this project.

Contributors: SNW, JF, AP, SB, LNS, and MC were responsible for conception and design. SNW, JF, DB, LM, and MC collected the data. SNW, AP, LNS, and MC analysed and interpreted data. SNW, JF, DB, LM, AP, SB, LNS, and MC drafted and revised the paper. AP and SNW carried out the statistical analysis. SNW, JF, DB, LM, SB, LNS, and MC were responsible for administrative, technical, and material support. SNW and LNS supervised the study. SNW is guarantor.

Funding: Center for Patient Safety, Dana-Farber Cancer Institute, Boston. SNW was also supported by a K08 Mentored Clinical Scientist Caree Development Award (1 K08 HS 11644) from the US Agency for Healthcare Research and Quality.

Competing interests: None declared.

Ethical approval: Dana-Farber Cancer Institute's institutional review board. Half of the centres required no safeguards around prescription writing at all.

\section{Study limitations}

Medication safety practices at sites that did not respond may differ from those that responded. Our results may overemphasise the perspective of pharmacy directors relative to other oncology clinicians. Similarly, respondents' characterisation of safety practices reflected their best-but potentially biased-judgments. We asked respondents to consider oral agents with considerable toxicity risks, but they may not have shared a common definition of oral chemotherapy. Finally, it may have been difficult for respondents to characterise medication practices in organisations with practice patterns that varied across clinicians and treatment regimens.

\section{Conclusions}

Our data indicate that prescribing, monitoring and coordination, pharmacy practices, and education of patients for oral chemotherapy vary substantially. Despite clinicians' concern about oral
1 O'Neill VJ,Twelves CJ. Oral cancer treatment: developments in chemotherapy and beyond. Br / Cancer2002;87:933-7.

2 DeMario DM, Ratain MJ. Oral chemotherapy: rationale and future directions. J Clin Oncol1998;16:2557-67.

3 Bedell $\mathrm{CH}$. A changing paradigm for cancer treatment: the advent of new oral chemotherapy agents. Clin I Oncol Nurs 2003;7(6 suppl):5-9.

4 Partridge AH,Avorn J,Wang PS,Winer EP. Adherence to therapy with oral antineoplastic agents. J Natl Cancer Inst 2002;94:652-61.

5 Sharma S. Patient selection for oral chemotherapy. Oncology 2001;15(1 suppl 2):33-5.

6 Birner A. Safe administration of oral chemotherapy. Clin J Oncol Nurs2003;7:158-62.

7 Faithfull S,Deery P. Implementation of capecitabine (Xeloda) in a cancer centre: UK experience. Eur I Oncol Nurs 2004;8(suppl 1):S54-62.

8 Griffin E. Safety considerations and safe handling of oral chemotherapy agents. Clin I Oncol Nurs 2003;7(6 suppl):25-9.

9 Hartigan K. Patient education: the cornerstone of successful oral chemotherapy treatment. Clin J Oncol Nurs 2003;7(6 suppl):21-4

10 Schulmeister L. Ten simple strategies to prevent chemotherapy errors. Clin JOncol Nurs2005;9:201-5.

11 Taylor JA,Winter L,Geyer LJ,Hawkins DS. Oral outpatient chemotherapy medication errors in children with acute lymphoblastic leukemia. Cancer2006;107:1400-6.

Accepted: 5 December 2006 